\title{
MITHILA'S 'FAKDE' ENCOMPASSES VARIOUS COLORS OF LIFE
}

\section{जीवन के विविध रंगों को समेटे हैं मिथिला के 'फैकड़े'}

\author{
Dr Niraj Kumar Mishra ${ }^{*}{ }^{\square}$ \\ *1 TM Bhagalpur University, Bihar, India
}

DOI: https://doi.org/10.29121/granthaalayah.v8.i10.2020.1617

Article Type: Research Article

Article Citation: Dr Niraj Kumar Mishra. (2020). MITHILA'S 'FAKDE' ENCOMPASSES VARIOUS COLORS OF LIFE. International Journal of Research -GRANTHAALAYAH, 8(10), 323-327. https://doi.org/10.29121/granthaa layah.v8.i10.2020.1617

Received Date: 18 September 2020

Accepted Date: 31 October 2020

Keywords:

जीवन

रंगों

मिथिला

\section{ABSTRACT}

English: Loktokis are particularly associated with the social life of a particular region and the language and culture of the region. Loktokis are the treasures of empirical knowledge. The facts that humans have interviewed through the ages are published through them. These are the sources of long-felt knowledge. The main objective is to publish a largely realized wisdom in a concise form. If the ideology of a caste has flowed from centuries, it is necessary to study the ethos of that caste.

Hindi: लोकोक्तियाँ किसी क्षेत्र विषेश के सामाजिक जीवन और वहाँ की भाषा और संस्कृति से खास तौर से जुड़ी होती हैं। लोकोक्तियाँ अनुभवसिद्ध ज्ञान की निधि हैं। मानव ने युगयुग से जिन तथ्यों का साक्षात्कार किया है, उनका प्रकाशन इनके माध्यम से होता है। ये चिरकालीन अनुभूत ज्ञान के सूत्र हैं। समास रूप में चिरसंचित अनुभूत ज्ञान राषि का प्रकाशन इसका प्रधान उद्देश्य है। शताब्दियों से किसी जाति की विचारधारा किस ओर प्रवाहित हुई है, यदि इसका दिग्दर्शन करना हो तो उस जाति की लोकोक्तियों का अध्ययन आवश्यक है।

\section{1. प्रस्तावना}

संसार का कोई भी क्षेत्र हो, चाहे वह विज्ञान हो, समाज हो, अध्यात्म हो अथवा साहित्य हो; अनुभव से वह परिपक्र और पुश्ट होता रहा है। किसी भी लोकभाषा में मौजूद इन लोकोक्तियों अथवा कहावतों ने सचेतक की भूमिका निभाई है। डॉ. अंजलि चैहान के अनुसार "विद्वानों ने कहावतों को ग्रामीणों का नीतिशास्त्र कहा है। इसी दृ्टि से कहावतें संसार की नीति साहित्य (विज्डम लिटरेचर) का प्रमुख अंग मानी जाती हैं। इन कहावतों में एक व्यापकता समाहित है। यह व्यापकता गागर में सागर के सहष है।"[1] संसार की अधिसंख्य बोलियों की अपनी लोकोक्तियाँ हैं। ये पढ़े-लिखे, विद्वान अथवा अनपढ़ लोगों की अभिव्यक्तियों में समान रूप से पायी जाती रही हैं। रामकृश्णमिषन विद्यापीठ के भूतपर्व हिन्दी अध्यापक केदारनाथ के अनुसार 'सम्पूर्ण एवं स्वतंत्र वाक्यों के प्रवाह को ही कहावत कहते है। जो बात समय या मौके पर कही जाती है, उसे लोकोक्ति या कहावत कहते हैं। लोकोक्ति का शाब्दिक अर्थ होता है- मनुष्य की उक्ति। अर्थात् मनुष्य द्वारा अनोखा या चमत्कारपूर्ण वाक्य या व्यवहार।'[2] यद्यपि हिन्दी में कहावतों को लोकोक्ति मानकर उसे अलंकार के अंतर्गत रखा गया है। 'पद्माभरण' में पद्माकर इस बात की पुश्टि भी करते हैं 'जहाँ लोक की कहनावति ठहराउ।' पर यह अत्यंत दुखद है कि जब-जब ये क्षेत्रिय बोलियाँ परिमार्जित होकर भाशा का स्वरूप ग्रहण करती हैं, तब एक अनावश्यक शहरीपन का शिकार हो जाती हैं। और ये ज्ञान सूत्र उपेक्षित होकर बाहर छूट जाते हैं। उदाहरण के लिए हिन्दी की क्षेत्रिय बोलियों में इन लोकोक्तियों का प्रयोग जिस खुलेपन के साथ होता है, वैसा साहित्य के स्तर पर नहीं होता। इन लोकोक्तियों की भूमिका रोजमर्रा की जिन्दगी में एक शिक्षक के जैसी है। घर के बड़े-बुजुर्ग, माँ, दादी-नानी बात-बात में इनका प्रयोग शिक्षा देने और अनुशासन बनाए रखने के लिए करती हैं। एक कहावत अथवा एक लोकोक्ति किसी कहानी अथवा घटना के शीर्षक की तरह होती हैं, जिसकी चर्चा होते ही एक घटना अपने नैतिक निष्कर्षों के साथ उपस्थित हो जाती है। डॉ. बासुदेव नन्दन प्रसाद के अनुसार भी- "लोकोक्ति के पीछे कोई कहानी या घटना होती है। उससे निकली बात बाद में लोगों की जुबान पर जब चल निकलती है, तब 'लोकोक्ति' हो जाती है।"[3] मनुश्य का यह स्वभाव है कि वह अपनी गलतियों से सावधान होता है और उससे सीख लेता है। उसी

(C) 2020 The Author(s). This is an open access article distributed under the terms of the Creative Commons Attribution License, which permits unrestricted use, distribution, and reproduction in any medium, provided the original author and source are credited. 


\section{जीवन के विविध रंगों को समेटे हैं मिथिला के 'फैकड़े'}

प्रकार वह अपने पूर्व के अच्छे कार्यों से प्रेरणा ग्रहण कर आगे भी उस राह पर चलने अथवा उन तरीकों को ग्रहण करने के प्रति उत्साहित रहता है। अंग्रेजी में लोकोक्ति के लिए 'एडेज' षब्द है। अंग्रेजी में 'फ्रेज' को गढ़ने का श्रेय बहुत कुछ शेक्सपीयर को दिया जाता है। उनके रचित साहित्य में लोकोक्तियों का भी प्रयोग अनेक स्थलों पर हुआ है। हिन्दी साहित्य में भी लोकोक्तियाँ यत्र-तत्र प्रयुक्त होती रही हैं। पर हिन्दी की उपभाशाओं में इनका प्रयोग धड़ल्ले से हुआ है। मैथिली भाशी लोगों की बोलचाल में इन लोकोक्तियों के अनोखे प्रयोग के दर्षन होते हैं। इस आलेख का मुख्य लक्ष्य मैथिली में प्रयुक्त लोकोक्तियों का अध्ययन कर सम्बद्ध समाज और मिथिला संस्कृति पर इनके प्रभावों का महत्वांकन करना है। मैथिली में इन्हें 'फैकड़ा' अथवा 'पढुआ' भी कहा जाता है। यदि मैथिली में प्रयुक्त इन फैकड़ों के उद्दव और प्रयोग का सूक्ष्मता से अध्ययन किया जाय तो मिथिला के समाज के बौद्धिक, सांस्कृतिक और तार्किक विकास का पता चलता है। प्रस्तुत आलेख में क्रम से इन फैकड़ों के अर्थ और इनकी उपयोगिता पर विचार होगा-

1) माय हेरे अँतड़ी, बौह हेरे पोटरी- इस लोकोक्ति के निर्माण के पीछे की धारणा यह है कि बेटे के प्रति माँ का स्नेह संसार के किसी भी अन्य व्यक्ति की तुलना में ज्यादा है; क्योंकि माँ अपने बच्चों को सारे कष्ट उठाकर बड़ा करती है। इस लोकोक्ति के पीछे की घटना यह है कि बेटा जब थककर घर लौटता है तो पत्नी जिसे वह सबसे अधिक प्यार करता है, वह सबसे पहले उसकी दिन भर की कमाई के बारे में जानकारी लेना चाहती है। पर माँ इसी चिंता में रहती है कि उसने दिन भर कुछ खाया-पीया कि नहीं ? परंतु मैथिली में इस फकरे का प्रयोग विविध प्रसंगों के लिए होता है। जैसे- यदि हम किसी उद्योग की स्थापना में विभित्र प्रकार के यन्तों को काफी मेहनत और उद्यम से उपलब्ध करते हैं, तो उत्पादन और लाभ के साथ-साथ हमें इस उद्योग में प्रयुक्त यन्त्रों की सुरक्षा की भी उतनी ही चिन्ता रहती है।

2) लाजे भाभौ बोलै नै, ढीठे भैंसुर छोड़ै नै- इसका अर्थ यह हुआ कि विनम्रता और लीहाज एक सीमा तक ही सही है, अन्यथा षोशण और प्रताड़ना झेलनी पर सकती है। मिथिला संस्कृति में भैंसुर पति के बड़े भाई को कहा जाता है। उससे बात करना तो दूर, उससे काफी दूरी बनाए रखने की परिवार में हिदायत है।

3) भैर घोर द्योर, भैंसुर से ठठ्ठा- मतलब यह कि हमउम्र और सहकर्मियों की पर्याप्त संख्या के बाद भी अपने सीनियर्स के साथ बिना वजह के महत्वहीन प्रष्नों पर बहस लड़ाना। इसका इषारा कार्य-स्थल पर अनुशासनहीनता के कारण गरिमा के नष्ट होने की ओर भी है।

4) घोर एंगना छाय सन, बौह लगै छन माय सन- योग्य संचालक के अभाव में कार्य स्थल पर अव्यवस्था छा जाती है; ठीक उसी प्रकार जैसे कुषल गृहिणी के नहीं होने पर घर-आँगन अस्त-व्यस्त हो जाता है।

5) बूढ़ सुग्गा पोस मानै छय- इसका अर्थ यह हुआ कि शिक्षा ग्रहण करने और दक्षता हासिल करने की एक उम्र होती है। उचित समय निकल जाने के बाद रूचि और अवसर दोनों कम हो जाते हैं। ठीक उसी प्रकार जैसे तोते की ज्यादा उम्र हो जाने के बाद उसे बहुत सी बातें सिखाना और पालतू बनाना मुष्किल हो जाता है।

6) कोड़क माऽर सहे भलनाऽर, फूलक माऽर आबे जुआऽर- रोज जिसे दुर्व्यवहार सहने की आदत हो जाती है, वह इसे ही अपना भाग्य समझकर षांत रहने लगता है। पर जिसे हमेषा प्यार और सम्मान ही मिला हो, वह थोड़ी सी झिड़की भी बर्दाश्त नहीं कर पाता है। इस लोकोक्ति के कई अर्थ हैं। जब हमारा जीवन दुख में ही गुजरता है तब हम बड़े से बड़ा अपमान भी अपनी किस्मत समझकर सह लेते हैं। पर सुख और सामथ्य के दिनों में अपनी षान में थोड़ी सी गुस्ताखी भी नहीं सह सकते।

7) जे बियैली से ललैली, बेटा ले पड़ोसिन गेली- इसका मतलब यह हुआ कि जो व्यक्ति किसी कार्य को अपनी मेहनत और कठिन परिश्रम से अत्यंत महत्व का बनाता है, वह इससे मिलने वाले लाभ को प्राप्त नहीं कर पाता और कोई दूसरा लाभ और यष पर मालिकाना हक जमा लेता है। यह फैकड़ा शायद मिथिला के उन लड़कों की हरकतों को देखते हुए अवश्य ही माताओं ने ईजाद किया होगा, जो माँ की जगह सासू माँ की भक्ति करने लगे होंगे।

8) जित्ता में गुह भात, मरला पर दूध भात-समाज की विचित्र परिपाटी है। जिन्हें जीवन-काल में सम्मान मिलना चाहिए उन्हें उपेक्षा झेलनी पड़ती है। किन्तु मरणोपरांत दिखावे के लिए उनका यषोगान किया जाता है। इसका एक अर्थ यह भी है कि समय रहते यदि हम किसी चीज की देखभाल ठीक से न करें तो बाद में कितनी भी कोशिष क्यों न की जाय उसका कोई लाभ नहीं।

9) जुड़े मियाँ क माँड़ नै, माँगे मियाँ ताड़ी- अपनी औकात का ध्यान रखे बिना बड़ी-बड़ी बातें करना। किसी भी योजना को अमलीजामा पहनाने के लिए कुछ आधारभूत चीजों की जरूरत होती है। बिना उसकी व्यवस्था किए सफलता के दावे करना अत्यंत हास्यास्पद है।

10) साँय बौह में झगड़ा, पंच भेल लबड़ा- किसी के घर के, अथवा एक दूसरे पर आश्रित लोगों के छोटे-मोटे झगड़े में नहीं पड़ना चाहिए वरना स्वयं ही बदनामी उठानी पड़ सकती है।

11) खाय के उपाय नै, ड्यौढी पर नाच- अपनी आर्थिक क्षमता से ज्यादा खर्च करने की प्रवृत्ति पर यह लोकेक्ति एक चुभता हुआ व्यंग है। मिथिला समाज धार्मिक-सामाजिक रूढियों से बुरी तरह प्रभावित रहा है। विवाह-श्राद्ध आदि पर दिखावे के लिए भोज करना और फिर कर्ज में दबकर बर्बाद हो जाना यहाँ के लोगों के लिए कोई नयी बात नहीं है।

12) चले क चेठा नै, गोदी म ठुनठुनाय- इसका सीधा अर्थ यह है कि छोटा बच्चा खुद से तो ठीक से चल भी नहीं पाता पर गोद से उतरकर खुद से चलने की जिद करता रहता है। पर इसका निहितार्थ बेहद प्रभावशाली है। यह वैसे लोगों की प्रवृत्ति पर एक व्यंग्य की तरह इस्तेमाल होता रहा है, जो दूसरों के किये-धरे पर पलकर 'मैं यह कर देता, मैं वैसा कर देता' की डींगे मारा करते हैं। पर जब मौका आता है तब किसी काम के साबित नहीं होते।

13) बाप छल पेट में, पूत गेल गया- किसी काम की षुरूआत भी नहीं हुई पर कल्पनाजीवी लोग अरमानों के महल खड़ा करने लगते हैं। प्रायः ऐसा देखा गया है कि आरामतलब लोग काफी उदारता से सपने देखा करते हैं। योजना बनाकर कार्यान्वित नहीं करना ऐसे लोगों की सामान्य आदत होती है। पर कल्पना के लोक में उड़ान भरने में कोई खर्च थोड़े ही है, वे बैठे-बैठे अपने हरवाहे के लिए भी अपने महल के पास ही एक छोटा सा घर दे देने की योजना भी बना डालते हैं। 
14) खस्सी के जान जाय, खवैया के सुआदे नय-कोई अपनी पूरी मेहनत और सारी सम्पत्ति परोपकार के कार्य में अथवा किसी सामाजिक-पारिवारिक कार्य में खर्च कर डालता है पर उपभोग करने वाले लोग उसमें भी मीन-मेख निकालने से बाज नहीं आते हैं। मिथिला के लोग ब्याह-श्राद्ध आदि में सामथ्य से ज्यादा खर्च कर देते हैं, फिर भी कमियाँ ढूँढने वाले अपने कृत्य से बाज नहीं आते हैं।

15) काँइख तरि अँचार, आ देखाबय पोथी के विचार- यह व्यंगोक्ति कथनी और करनी के अंतर पर चोट करती है। दूसरों के सामने तो लोग ऊँचे आदर्षों की बात करते हैं, पर अंतर में बुरे भावों (ईश्र्या-द्वेश) को छुपाये रखते हैं। खड़ी बोली हिन्दी में इसका एक समानार्थी कहावत 'मुँह में राम बगल में छुरी' है।

16) जेकर माय मरल, तेक्कर पत्ता पर भाते नै- जिसके त्याग से यह महान कार्य अथवा उद्देश्य आज पूर्ण हुआ है, उसकी तो चर्चा भी यहाँ कोई करने वाला नहीं है। सभी अपनी पीठ थपथपाने पर लगे हैं।

17) मसुरक दायल में इमली के खट्टा, भाय-बहिन में हँस्सी ठठा- स्थान विषेश और परिस्थिति को ध्यान में रखकर किया जाने वाला आचरण ही उचित और मान्य होता है। अन्यथा कोई कृत्य अथवा विचार अनैतिक और अप्रासंगिक सिद्ध हो जाता है। कभी-कभी कुछ बातें अथवा कार्य परिवार और समाज की संस्कृति के प्रतिकूल बैठने लगती है। जैसे किसी धार्मिक परिचर्चा के बीच फिल्मी गाने गुनगुनाने लगना उसी प्रकार अनर्गल और अनैतिक लगने लगता है, जैसे भाई बहन के बीच अभद्र बातचीत। मैथिली का यह फैकड़ा परिवार में रिश्तों की मर्यादा को बचाये रखने के लिए सचेतक के रूप में प्रयुक्त होता रहा है, और आज भी घर में दादी, नानी और माँ के मुख से सुना जा सकता है।

18) भुसकोल विद्यार्थी के गत्ता मोट- किसी कमी को ढँकने के लिए जब लोग बाहरी टीप-टॉप का सहारा लेने लगते हैं, तब सामाजिकों के व्यंग्य के शिकार बन जाते हैं। प्रस्तुत फैकड़ा इसी उद्देष्य से रचा गया लगता है।

19) कानय के मोन छल, त आँख में गरल खुट्टी- समाज विभिन्न प्रकार और विचार वाले व्यक्तियों का समूह होता है। एक-दूसरे की आपसी सहभगिता किसी भी समाज के निर्माण की आवश्यक शर्त होती है। प्रत्येक व्यक्ति अपने सुख-दुख में अन्य के सहयोग और शिरकत की अपेक्षा रखता है। प्रस्तुत लोकोक्ति वैसे लोगों पर एक व्यंग्य है, जो स्वयं जरूरत पड़ने पर दूसरों की सहायता ले लेते हैं, पर अपनी बारी आने पर बहाने बनाने लगते हैं।

20) अनकर धान पाबी, त अस्सी मन तौलाबी- मुफ्त में मिलने वाली चीजों के लिए जी से लार टपकने लगना सामान्य सी बात है। चूँकि इसमें पैसे का व्यय नहीं होता है, अतः लोग ज्यादा से ज्यादा खुद हडपने की ताक में रहते हैं

21) जे पूत परदेस गेल, देव पितर सब स गेल- क्षेत्र विषेश की एक संस्कृति होती है। मनुष्य के सभ्य बने रहने, परिवार और समाज की परिपाटियों के पालन और संरक्षण के लिए यही संस्कृति कुछ अनुशासनात्मक नियमों को सहेज कर चलती है। जब तक व्यक्ति उस सांस्कृतिक परिवेष में जीता है, उन नियमों को मानने के लिए बाध्य होता है। परन्तु एक परदेशी के लिए इन बातों को मानने की कोई बाध्यता नहीं होती है।

22) फेर की नाढ़ो बेल तोर जैती, बेलक मायर पटापट खैती- इस लोकोक्ति का अर्थ यह है कि विपत्ति में पड़े व्यक्ति को तुरत कठिन राह का चुनाव नहीं कर लेना चाहिए। इससे कष्ट के बढ़ जाने की संभावना रहती है और व्यक्ति कठिनतम परिस्थितियों में फँस सकता है। सर मुंडाये व्यक्ति को मिथिला में 'नाढ़ा' कहा जाता है। चूँकि यह प्रयोग किसी स्त्री के लिए हुआ है अतः उसे 'नाढ़ो' से संबोधित किया गया है। नाढो बेल के गाछ के नीचे क्या गयी, बेल के फल और टहनियों की मार ऐसी पड़ी कि फिर कभी नहीं गयी।

23) अल्हुआ सुथनी घूरा बिगाड़े, कुटनी मौगी टोला बिगाड़े- गलत लोगों का प्रवेष व्यवस्था को बिगाड़ देता है। जाड़े के दिनों में गाँवों में अलाव जलाकर षाम से ही लोग तापने के लिए बैठ जाते हैं। मिथिला में बच्चे अल्हुआ (षकरकंद) और सुथनी जैसे देहाती फलों को अलाव जिसे मिथिला में 'घूरा' कहा जाता है, में छिपाकर डाल देते हैं। ये फल गर्म होकर पानी छोड़ने लगते हैं और 'घूरा' को बुझा देते हैं। ठीक उसी प्रकार मिथिला के गाँवों में नमक-मिर्च लगाकर इधर की बात उधर करके दूसरों को आपस में लड़वाने वाली महिला को 'कुटनी' कहा जाता है। ये औरतें गाँव की षांति व्यवस्था में खलल उत्पन्न करने के कारण खासी बदनाम हैं। इस फैकड़ा का प्रयोग विविध प्रसंगों के लिए होता है।

24) बाबा जी के बेल हाथे-हाथ गेल- समाज में सीधे-सादे और कमजोर लोगों के साथ चालाक और दबंग लोग ज्यादती करने से कभी बाज नहीं आते हैं। ऐसे लोग कमजोर और जरूरतमंद लोगों का हक मारकर समाज में घृणित असमानता को जन्म देते हैं।

25) मेहनत करे मुर्गी, आ अंडा खाय दरोगा- समानता के तमाम प्रयासों और दावों के बावजूद आज भी हमारे समाज में छोटा-बड़ा, गरीबअमीर का भेदभाव खत्म नहीं हो पाया है। हमारा समाज इस आधुनिक युग में भी मध्य वर्ग की 'दबंगई' से त्रस्त है। आज की तारीख में राजनेता, अधिकारी, व्यापारी, दलाल आदि अलग-अलग ढंग से सामान्य मेहनतकष का षोशण करते हैं। मजदूर दिन भर काम करता है और अधिकारी और ठेकेदार मलाई काटते हैं। किसान जी तोड़ मेहनत कर फसल उपजाता है, पर बिचैलिये और व्यापारी सारा फायदा लेकर किसानों को आत्महत्या पर मजबूर कर देते हैं। आज के दौर के लिए यह उक्ति काफी प्रासंगिक लगती है।

26) हरबरी क बियाह म कनपट्टी पर सिन्दूर- किसी भी कार्य को ढंग से पूर्ण करने के लिए उचित समय देना ही पड़ता है। ज्यादा जल्दबाजी के कारण काम बिगड़ भी जाता है और परिहास का विषय बन जाता है। ठीक उसी प्रकार जैसे हड़बड़ी की शादी में दुल्हा सिन्दूर माँग के बदले कनपटी में डाल देता है।

27) अघायल बगुला के पोठी तीत- जब व्यक्ति रोटी, कपड़ा और मकान तीनों स्तरों पर तृप्त हो जाता है तो वह हर चीज में नुक्स निकालने लगता है, कमियाँ ढूँढने लगता है।

28) मैर मैर काटौं मालहिं जाय- इसका अर्थ है काफी परिश्रम से उपार्जित धन अथवा सम्पत्ति का महत्वहीन कार्यों में व्यय हो जाना या उसका सही मूल्य नहीं मिलना। 
29) राम भरोसे हिन्दु होटल- यह लोकोक्ति मिथिला में काफी लोकप्रिय है। यह वहाँ के समाज में व्याप्त गरीबी से उत्पन्न अवसाद और अनिश्रय से ईश्वर के भरोसे निपटने की प्रवृति को अभिव्यक्त करता है। यह मैथिलों के मिजाज और उनके बिन्दास जीवन षैली की ओर भी इशारा करता है।

30) हाथ गोर म दम नै, आ ककरो स कम नै- यह उक्ति उन लोगों के लिए कही गई हैं जो शारीरिक रूप से कमजोर होने के बाद भी दिमागी स्तर पर काफी मजबूत होने के कारण समाज में प्रतिष्ठा प्राप्त करते हैं। कलाकार, शिक्षक, कवि, दार्शनिक, पंडित, तान्त्रिक आदि इस वर्ग में आते हैं।

31) जे सब सौं छोट, से उनचास हाथ- मिथिला में गुणों के बखान में अतिषयोक्ति अलंकार की भारतीय परंपरा को लोकोक्तियों में भी स्थान दिया गया है। अपने लोगों अथवा अपनी चीजों की प्रषंसा बढा-चढा करने में यहाँ के लोग अत्यन्त कुषल हैं।

32) अप्पन दही केयो खट्टा कहै छै- हिन्दी की लगभग सभी उपभाशाओं में यह लोकोक्ति मोजूद है। यह लगभग सभी सन्दर्भों में अपनी सार्थकता सिद्ध करती है। इसका सीधा अर्थ यह है कि लोग अपनी चीजों अथवा अपनों की हमेषा तारीफ ही करना चाहते हैं। चाहे उनमें कितनी भी बुराईयाँ क्यों न हो।

33) करनी देखियोन मरनी बेर- हमारे धार्मिक ग्रन्थों में मृत्यु के समय होने वाले कष्टों को व्यक्ति के द्वारा जीवन काल में किए गए बुरे कर्मों से जोड़कर देखा जाता है। इसके अलावा मृतक के अच्छे कर्म उसे मरणोपरांत यश प्रदान करते हैं। यही वजह कि जननायक, लेखक, कवि और कलाकार मृत्यु के बाद भी लोगों के दिलों में जीवित रहते हैं।

34) खो मंगला पड़ल रह- यह फैकड़ा समपूर्ण मिथिला क्षेत्र में लोगों की जुबान पर रहता है। इसमें एक सकारात्मक निष्क्रियता का भाव सत्रिहित है। यदि व्यक्ति की सक्रिय भागीदारी से किसी प्रकार की अशांति अथवा विवाद की संभावना हो तो उस परिस्थिति में निष्क्रिय होकर पड़ जाना ही समझदारी है।

35) जेत्ते क बौह नै ओत्ते क लहठी- जिस कार्य में फायदा कम और खर्च ज्यादा हो उसे कार्यान्वित नहीं करने में ही फायदा है। अन्यथा व्यापार के घाटे से उबरना मुश्किल होगा। अतः सोच-समझकर ही निवेश करना चाहिए।

36) जे भोज नै करै, से दायल बडु पिबै- जो खुद कोई काम नहीं करता अथवा कोइ आयोजन नहीं करता वह दूसरों को इस निमित्त नाहक ही ढेर सारे सलाह और चेतावनियाँ देता फिरता है। यह उक्ति कंजूस और चालाक लोगों की कार्यशेली पर व्यंग्य है।

37) हेहरी गे हेहरी कोना रहै छें, लायत जुत्ता खाय छौं भने रहै छौं- यह लोकोक्ति मिथिला की उन स्त्रियों के लिए प्रचलन में है जो अपनी बुरी आदतों के कारण समाज में अपमानित होती हैं, पर अपनी आदतें ठीक नहीं करती हैं।

38) धरमे धाँधड़ पापे बिरधी- कोई भी सत्कार्य अनेक अवरोधों के बाद भी ठीक से पूर्ण नहीं होता है। अच्छे, साधु स्वभाव के लोग हमेशा कष्ट ही पाते हैं। ठीक इसके विपरीत बुरे और दुश्ट स्वभाव के लोगों की तरक्की हर युग में देखी गयी है।

39) चलनी दुसलक सूप के, जेकरा सहस्सर छेद- ऐसा प्रायः देखा गया है कि जो लोग स्वयं अवगुण की खान होते हैं, चरित्रहीन होते हैंय दूसरों की कमियाँ ढूँढते रहते हैं।

इसके अलावा सैकड़ों ऐसी लोकोक्तियाँ हैं जो संग्रह के अभाव में इस आलेख के अन्तर्गत नहीं प्रकाशित की जा सकी हैं। कई उक्तियाँ समान अर्थ वाली हैं। जैसे-'माय बाप करे कुटान पिसान बेटा के नाम दुर्गादास' और 'बाप के नाम लत्ती फत्ती बेटा के नाम कदीमा' का एक ही अर्थ है। उसी प्रकार 'पाइन मे माछ नौ नौ कुटिया बखरा' और 'बाप छल पेट म पूत गेल गया' समान अर्थ वाले हैं। इसके अलावा 'चले त ठाँव चैढ क पादी नै त पादने पादने भागी' जैसी उक्यिएँ शिक्षा के साथ-साथ ग्रामीणों की आपसी बातचीत में हास्य रस का भी संचार करती हैं। कुछ अन्य उक्तियाँ जैसे'देखले दरभंगा अ चिन्हले चपरासी', मूर्खक लाठी बिच्चे कपार, पेट में खर नै सिंग में तेल इत्यादि अक्सर सुनने को मिल जाती हैं। एक-आध आधुनिक काल से प्रभावित उक्तियाँ जैसे-माय बाप बिसैर गेलों त बौह भेल फैमिली' प्रासंगिक हैं। छोटे आकार के इन वाक्यों में गूढ अर्थ समाहित होते हैं। इनमें व्यंग्य, मनोरंजन और शिक्षा, इन तीनों उद्देष्यों की पूत्रि होती है। डॉ. अंजलि चैहान के अनुसार "कहावतें सूत्र प्रणाली होती हैं। उनमें भावों की गंभीरता व मार्मिकता पहेली से अधिक गहरी और भाषा की शब्द -शक्ति लक्षण के सह्धष थोड़े में बहुत अर्थ की गंभीरता रहती है।"[4]

यह बात अत्यन्त दु:खद है कि मैथिली साहित्य में इन लोकोक्तियों की उपेक्षा की गई है। साहित्य में व्यंग्य के महत्व को ध्यान में रखते हुए इन फैकड़ों' का सुन्दर प्रयोग किया जा सकता है। क्योंकि इनमें से अधिकतर 'व्यंग्य' के रूप में ही हैं। व्यंग्य भाषा के अपने तेवर होते हैं, अपने अंदाज होते हैं। व्यंग्य की भाषा शास्त्र की भाषा नहीं होती, वह लोक की भाषा होती है। डॉ. श्यामसुंदर घोश लिखते हैं 'व्यंग्य लेखक की भाषा में धार और नोक दोनों जरूरी हैं। कभी नष्तर लगाता है, तो कभी खंजर चुभोता है।"[5] इन फैकड़ों को यदि सामान्य बोलचाल के स्तर से उठाकर साहित्य में पिरोया जाय तो व्यंग्य नई ऊँचाइयों को छुएगा, इसमें लेषमात्र भी संदेह नहीं है। खैर, साहित्यकारों ने इन्हें भले ही भुला दिया हो, पर ग्रामीणों के वार्तालाप में आज भी इनका बेधरक प्रयोग मिथिला की संस्कृति को जीवंत बनाए हुए है।

\section{SOURCES OF FUNDING}

None.

\section{CONFLICT OF INTEREST}

None. 


\section{डॉ. नीरज कुमार मिश्र}

\section{ACKNOWLEDGMENT}

None.

\section{REFERENCES}

[1] चैहान, डॉ. अंजलिय हरदोईः सांस्कृतिक गजेटियर, वाणी प्रकाषन, पृ0 286

[2] केदारनाथ, सामान्य हिन्दी व्याकरण और रचना, पृ० 210

[3] प्रसाद, डॉ. बासुदेव नन्दनय आधुनिक हिन्दी व्याकरण एवं रचना, पृ0 265

[4] चैहान, डॉ. अंजलिय हरदोईः सांस्कृतिक गजेटियर, वाणी प्रकाषन, पृ0 286

[5] घोश, डॉ. श्यामसुंदरय व्यंग्य क्या व्यंग्य क्यों, पृ० 119 